\title{
Actinomycetoma due to Actinomadura madurae: A therapeutic challenge. Case report
}

\author{
Ana Gabriela Fuentes-Nava ${ }^{1}$, Leonel Fierro-Arias ${ }^{1}$, Javier Araiza ${ }^{2}$, Mariana Ivonne Benitez- \\ Barradas $^{3}$, Hugo Eduardo Peláez González ${ }^{3}$, Alexandro Bonifaz ${ }^{1,2}$
}

${ }^{1}$ Department of Dermatology, Hospital General de México. "Dr. Eduardo Liceaga", Mexico City, Mexico, ${ }^{2}$ Department of Mycology, Hospital General de México. "Dr. Eduardo Liceaga”, Mexico City, Mexico, ${ }^{3}$ Department of Imagenology, Hospital General de México. "Dr. Eduardo Liceaga", Mexico City, Mexico

Corresponding author: Dr. Alexandro Bonifaz, E-mail:a_bonifaz@yahoo.com.mx

\begin{abstract}
Mycetoma is a chronic granulomatous infection that develops after traumatic inoculation of the skin with either true fungi (eumycetoma) or aerobic actinomycetes (actinomycetoma). Can be found in geographic areas in close proximity to the Tropic of Cancer also known as "mycetoma belt", the predominant climate of this region is subtropical and dry tropical. Actinomycetomas caused by Actinomadura spp. occur in drier areas with low relative humidity. We report a case in a 45 yearsold female, with a 9-years history of a progressive inflammatory tumor, sinuses tracts and granules on her left sole. She was treated with antibiotics and amputation without improvement. Actinomycetoma due to A. madurae was confirmed and successfully treated with a combination therapy that included streptomycin plus trimethoprim/sulfamethoxazol.
\end{abstract}

Key words: Mycetoma; Actinomicetoma; A. madurae; Streptomycin; Trimethoprim/sulfamethoxazole

\section{INTRODUCTION}

Mycetoma is a chronic, granulomatous, subcutaneous, inflammatory disease caused by true fungi (eumycetoma) or filamentous bacteria (actinomycetoma). Commonly affects adults, and the most common affected site are the feet. The characteristic clinical triad is tumefaction, draining sinuses and discharging grains or granules. It is caused by exogenous traumatic inoculation therefore, it is considered an infection by implantation. The global geographical distribution is $60 \%$ for actinomycetomas and $40 \%$ for eumycetomas $[1,2]$. It exists throughout the world, especially in intertropical countries like Asia, Africa and America. Eumycetomas predominate in Africa and Asia, and actinomycetomas in Latin America. In Mexico mycetomas are mainly actinomycetoma (98\%); 86\% are caused by Nocardia spp., of which $71 \%$ are due to $N$. brasiliensis. A. madurae is observed in $10 \%$ [3].

\section{CASE REPORT}

45 years-old woman native of México and resident of Waukegan, Illinois. Without chronic degenerative diseases diagnosed. In her clinical history must be noted that she made annual family visits to the state of Guerrero (endemic state of mycetoma), where there were houses built with adobe with dirt floors. In her pathological personal history she had a 9 years history of a progressive inflammatory tumor associated with sinuses tracts and tumefaction on her left sole. A surgical amputation of the $5^{\text {th }}$ metatarsal of the left foot in 2009 was made and she received previous treatments with trimethoprim/sulfamethoxazol [TMP/ SMX] and diaminodiphenyl sulfone [DDS] for 2 years without clinical improvement (Figs. la and lb).

She went to the dermatology service of the Hospital General de México in August 2017 where direct examination with potassium hydroxide $[\mathrm{KOH}]$ biopsy, image studies and microbiological cultures were made.

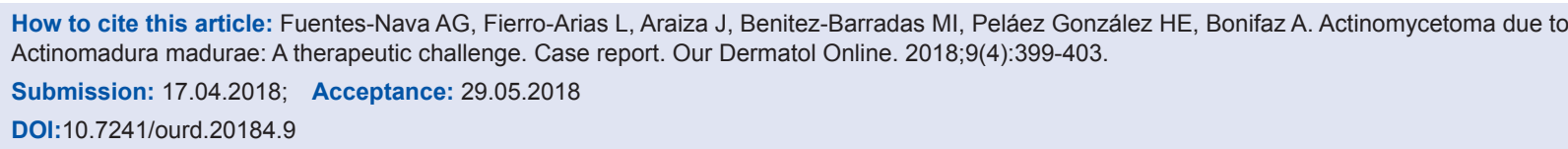


In direct examination two large, multi-lobed grains with size of $3 \mathrm{~mm}$ were observed (Fig. 2). Skin biopsy showed a granulomatous suppurative process with basophilic grains (Fig. 3). Microbiological cultures were positive, and Actinomadura madurae was identified.

X-rays of the foot showed an increase in soft tissue volume and in bone structures, lytic lesions, thickening of the cortex and cavities (Fig. 4). Finally in a high resolution computerized axial tomography the lytic lesions were confirmed as well as total absence of the $5^{\text {th }}$ metatarsian by previous amputation (Fig. 5).

With all the data obtained, the confirmed diagnosis was actinomycetoma due to Actinomadura madurae. We continue treatment with streptomycin $1 \mathrm{~g}$ three times a week to completing $50 \mathrm{~g}$., plus TMP/SMX 160/800 mg. The audiometries previous, during and at the end of the treatment were normal.

Prior to the study, patient gave written consent to the examination and biopsy after having been informed about the procedure.
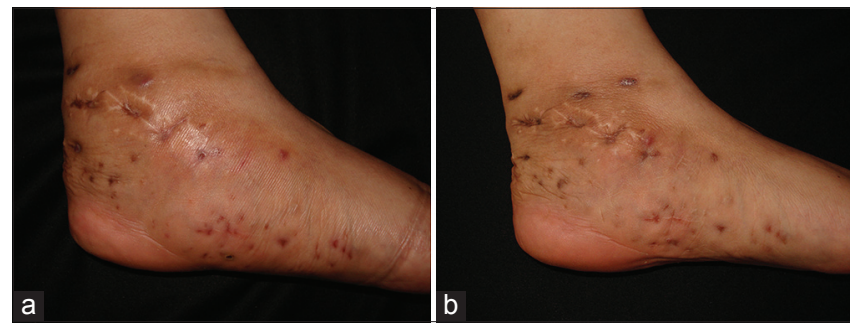

Figure 1: (a) Actinomycetoma baseline. (b) Actinomycetoma after 1 year and 8 months of treatment.

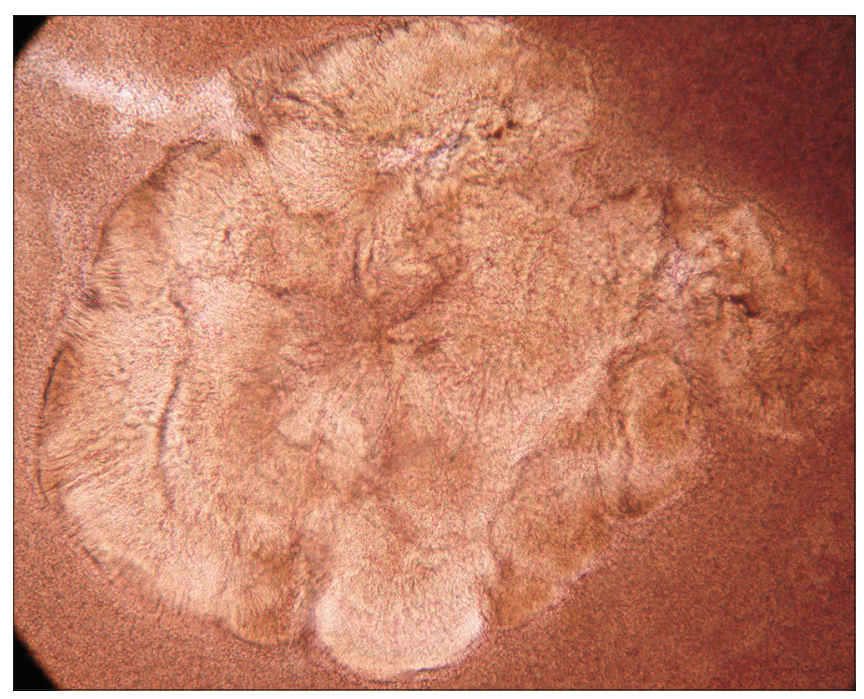

Figure 2: Large, multi-lobed grain with a caratographic, polylobulated and oval shape, characteristic of $A$. madurae. $(\mathrm{KOH}, 10 \mathrm{x})$.

\section{DISCUSSION}

On $28^{\text {th }}$ May 2016, mycetoma was recognized as a neglected tropical disease by the World Health Organization [3]. Mycetomas caused by A. madurae occur in drier areas with low relative humidity. Three main regions of A. madurae have been noted in México: The western center (Guanajuato, Michoacán, Jalisco and Querétaro), the southern center (Puebla, Oaxaca and northeast of Guerrero) and west of Hidalgo [1,2,4-7].

In 2016, the First Argentine Conference of Mycetomas was organized in Santiago del Estero, where a total of 159 thus far unpublished cases were presented. There was remarkable that actinomycetoma cases due to A. madurae are now slightly more frequent. It is important to mention that these cases are predominantly accounting for $55-70 \%$ of actinomycetoma cases; different from what is reported in Mexico $[3,4]$.

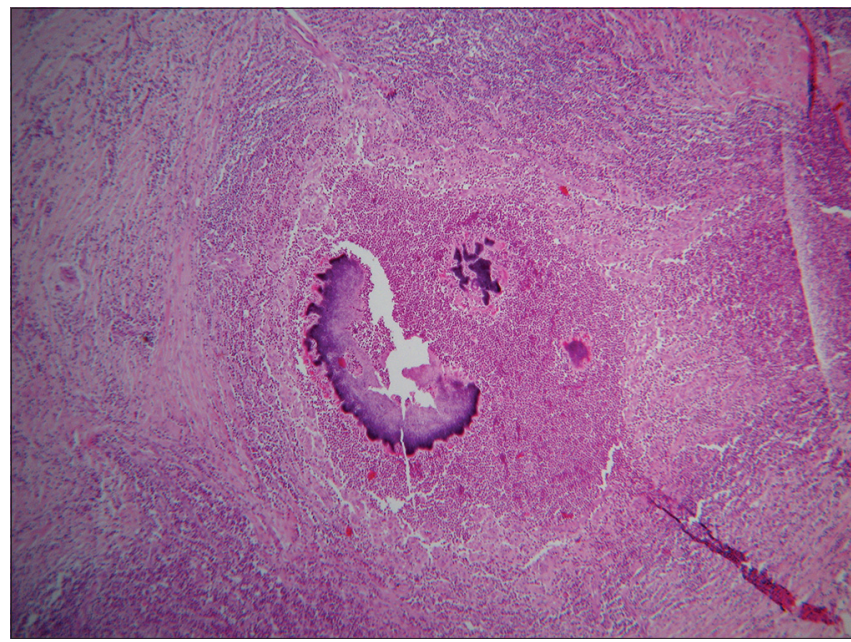

Figures 3: Granulomatous suppurative process with basophilic grain (H\&E, 10X).

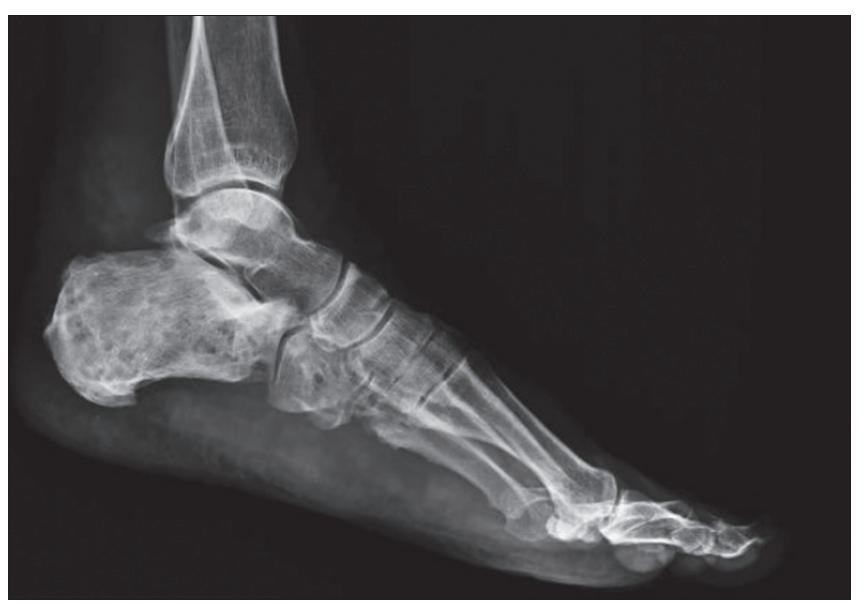

Figure 4: Radiographs of the foot showed lytic lesions, thickening of the cortex, cavities and mild soft tissue swelling. 
One of the most notable difference between mycetomas by A. madurae and by N. brasiliensis or by others agents is the predominance of A. madurae in females, a hormonal influence has been suggested; while the other mycetomas are more commonly reported in males (4:1), in the case of A. madurae the relationship is $2: 1[1,6-8]$.

Mycetoma by A. madurae is located mostly in the middle part of the foot affecting mainly the plant, unlike the mycetomas by Nocardia sp. that generally affect the lower third of the leg and malleolar region $[1,5,6]$. Other locations are extremely rare. Two cases have been reported in oral cavity, possibly in relation to the use of wood sticks for brushing teeth in many Afro-Asian communities [9].

Clinically they are more voluminous, of hard consistency, with few sinuses, a woody appearance and can resemble tumor processes of another origin. Unlike those produced by Nocardia spp. present a more inflammatory aspect, with numerous fistulas that exude a serous or purulent fluid and are generally of greater extension $[1,5,8]$.

A. madurae produces white and soft grains that can be identify through direct examination or with the naked eye, due to its large size and cartographic edges $[1,8]$. It grows slowly in about 1 month at $37^{\circ} \mathrm{C}$ on Sabouraud dextrose agar without antibiotics or in LowensteinJensen agar. The colonies are folded or cerebriform, with waxy aspect and yellow, white, pink or red color $[1,5,8]$.

The histopathology of mycetoma is very similar to the rest of subcutaneous mycoses, presents exulceration of the epidermis, pseudoepitheliomatous hyperplasia, fistula formation, dense inflammatory infiltrate composed of polymorphonuclears, lymphocytes, plasma cells and histiocytes with formation of giant cells, which give rise to a suppurative granuloma, with vascular proliferation, damage to the vessel walls and fibrous tissue. In the microabscesses of PMN are located the "grains". [8]

The grain of Actinomadura madurae measures from 1 to $20 \mathrm{~mm}$, with a caratographic, polylobulated and oval shape; clear in the center, dense purple in the periphery with with irregular and eosinophilic edges $[1,5]$. The tissue may be very friable if the biopsy is taken in an exulcerated or fistulous area, and there is a risk of not finding the grain if it is taken in a fibrous zone $[8,10,11]$.
There are some especially osteophilic agents, among them A. madurae, N. brasiliensis and M. mycetomatis. Most frequent alterations are periostitis, osteitis, osteofibrosis and osteolysis, with formation of cavities or osteophyte-holes "geodos" $[6,12]$. X-rays and tomographies are essential to indicate the degree of bone involvement. Computed helical tomography and MRI allows measuring the affected area and locating the specific damage (visceral and vascular). The recently described "dot in circle sign", a central tiny hypointense focus (Fig. 6) [13] on magnetic resonance imaging (MRI) is easy to recognize and specifc to this condition and establishes the role of MRI in early diagnosis [14].

The lytic lesions or cavities are due to replacement of bone tissue and marrow by masses of A. madurae grains. Those image findings are not specific and

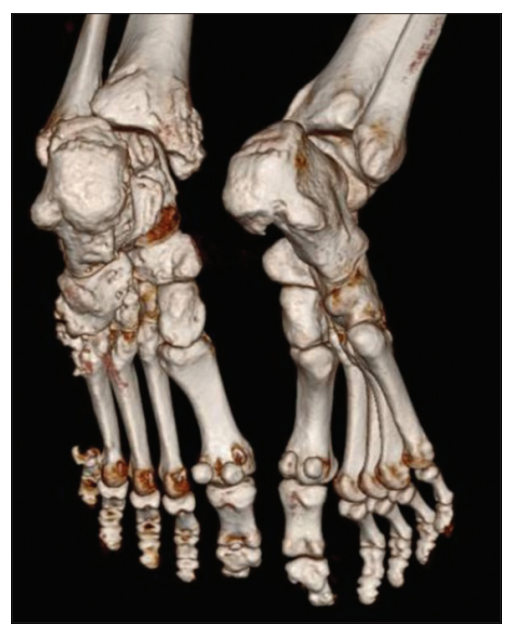

Figure 5: High resolution computerized axial tomography with many lytic lesions, thickening of the cortex as well as total absence of the 5 th metatarsian by previous amputation.

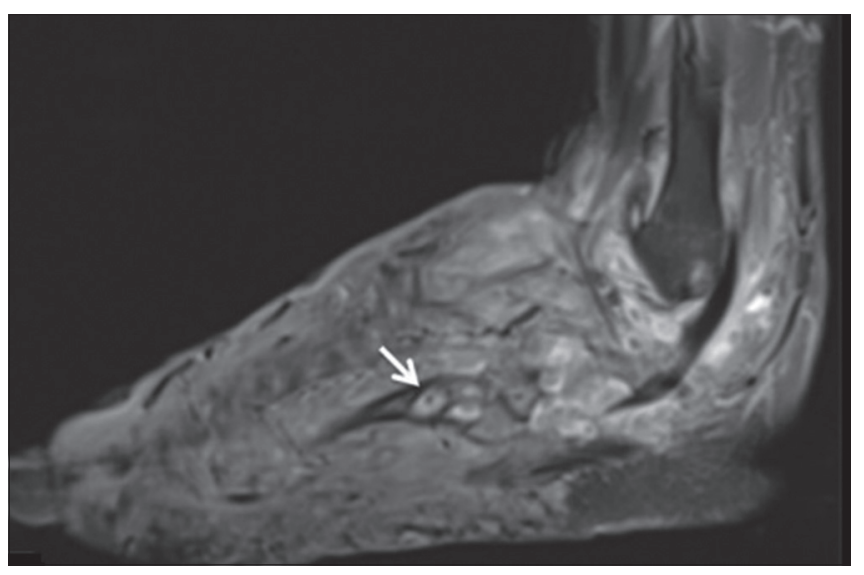

Figure 6: MRI T2 show inflammatory changes with multiple soft tissue and osseus small hyperintense lesions with peripheral hypointenserim corresponding to mycetoma grains (yellow arrows). Few of them showing "dot in circle" sign (thicker white arrow). 
cannot differentiate mycetoma from chronic bacterial osteomyelitis, granulomas, soft tissue tumors, bone tuberculosis and cold abscesses. On the contrary, the small ovoid-shape, low-signal lesions that we observed on T1- and T2-weighted images are more helpful in the diagnosis of actinomycetoma, since they are present in $80 \%$ of the cases [12].

The studies of susceptibility in Actinomadura madurae are not standardized, although low activity to penicillin, cephalosporins and trimethoprim-sulfamethoxazole has been described. In vitro A. madurae is sensitive to amikacin, TMP-SMX, linezolid and ciprofloxacin [15]. Therefore, it has been suggested that treatment should be combined. The best results have been with streptomycin (1 g once daily in adults; $20 \mathrm{mg} /$ $\mathrm{kg}$ once daily in children) until a total dose of $50 \mathrm{~g}$. is reached in combination with TMP-SMX or DDS. During the treatment with streptomycin otic function should be monitored. Also good response has been reported with ciprofloxacin in resistant cases. Despite the efforts, a good number of cases do not respond to treatment $[1,5,6,13]$. Alternatives for A madurae include streptomycin plus oral clofazimine $(100 \mathrm{mg}$ once daily), oral rifampicin (300 mg twice daily), oral tetracycline (1 g once daily), oral isoniazid (300-600 mg once daily), and oral minocycline (100 mg twice daily; also effective for A pelletieri) $[8,13,16]$.

Surgery is contraindicated in the actinomycetomas, since in most of these the process continues in the stump despite large surgical margins, and may even lead to lymphatic or hematogenous dissemination worsening the prognosis $[4-6,13,17]$.

The criteria used to guide the discontinuation of initial therapy for any mycetoma include a decrease in the volume of the lesion, closure of sinuses, 3 consecutive negative monthly cultures, image studies showing bone regeneration, lack of echoes and cavities on echography, and absence of grains on examination of fine-needle aspirates. After the initial treatment protocol is finished, most experts recommend continuing treatment with DDS 100 to $300 \mathrm{mg}$ once daily for several years to prevent recurrence $[6,13,14]$.

We present one case of a woman who according to the clinical characteristics had an eumycetoma. She was previously treated with TMP/SMX plus DDS for 2 years without clinical improvement. In our Institution we extended the diagnostic protocol and actinomycetoma due by A. madurae was confirmed. So we added streptomycin $1 \mathrm{~g}$ per day and suspended DDS. Currently our patient has significant improvement with a decrease in volume of the lesion, no more sinuses and negative cultures. Thereby demonstrating the benefit of combination therapy with streptomycin in actinomycetomas due by A. madurae.

The authors have no grants or other assistance and declare that they have no conflict of interest.

\section{CONSENT}

The examination of the patient was conducted according to the Declaration of Helsinki principles.

\section{REFERENCES}

1. Arenas R, Araiza J, Asz-Sigall, Bonifaz A, Castrillón-Rivera L, Dávila del Real MR, et al. Micetomas. Monogr Dermatol 2006;19:24-9.

2. López-Martínez R, Méndez-Tóvar LJ, Bonifaz A, Arenas R, Mayorga J, Welsh O, et al. Actualización de la epidemiología del micetoma en México. Revisión de 3,933 casos. Gac Méd Méx. 2013;149:586-92.

3. Bonifaz A, Tirado-Sánchez A, Calderón L, Saúl A, Araiza J, Hernández M, et al. Mycetoma: experience of 482 cases in a single center in Mexico. PLoS Negl Trop Dis. 2014;21:e3102.

4. Van de Sande W, Fahal A, Ahmed SA, Serrano JA, Bonifaz A, Zijlstra E. Closing the mycetoma knowledge gap. Med Mycol. 2018;56(suppl. 1):153-64.

5. Arenas R, Fernandez Martinez RF, Torres-Guerrero E, Garcia C. Actinomycetoma: an update on diagnosis and treatment. Cutis. 2017;99:E11-5.

6. Micetoma en: Bonifaz A. Micología médica básica. 5 ed. México: Mc Graw Hill;2015. P219-27.

7. Lavalle-Aguilar P, Padilla-Desgarennes MC, Pérez-Gutiérrez J, Rivera I, Reynoso-Rangel S. Micetomas por Actinomadura madurae en México. Rev Cent Dermatol Pascua. 2000;9:1.

8. Ahmed AA, Van de Sande W, Fahal AH. Mycetoma laboratory diagnosis: Review article. PLoS Negl Trop Dis. 2017;11:e0005638.

9. Mohamed el SW, Mohamed el NA, Yousif Bel D, Fahal AH. Tongue actinomycetoma due to Actinomadura madurae: a rare clinical presentation. J Oral Maxillofac Surg. 2012;70):e622-4.

10. Relhan V, Mahajan K, Agarwal P, Garg VK. Mycetoma: An Update. Indian J Dermatol. 2017;62:332-40.

11. Jerez R, Schafer F, Fich F, García P, León P, González S. Actinomycotic mycetoma due to Actinomadura madurae. Rev Chilena Infectol. 2012;29:459-63.

12. Ispoglou SS, Zormpala A, Androulaki A, Sipsas NV. Madura foot due to Actinomadura madurae: imaging appearance. Clin Imaging. 2003;27:233-5.

13. Jain V, Makwana GE, Bahri N, Mathur MK. The "Dot in Circle" Sign on MRI in Maduramycosis: A Characteristic Finding. J Clinical Imaging Sci. 2012;2:66.

14. White EA, Patel DB, Forrester DM, Gottsegen CJ, O'Rourke E, Holtom P, et al. Madura foot: two case reports, review of the literature, and new developments with clinical correlation. Skeletal Radiol. 2014;43:547-53.

15. Mencarini J, Antonelli A, Scoccianti G, Bartolini L, Roselli G, Capanna R, et al. Madura foot in Europe: diagnosis of an autochthonous case by molecular approach and review of the literature. New Microbiol. 2016;39:156-9. 
www.odermatol.com

16. Yeh I, Dhanireddy S. Madura foot caused by Actinomadura madurae in a pregnant woman. Arch Dermatol. 2010;146:1189-90.

17. Buonfrate D, Gobbi F, Angheben A, Marocco S, Farina C, Van Den Ende J, et al. Autochthonous cases of mycetoma in Europe: report of two cases and review of literature. PLoS One. 2014;9:e100590.
Copyright by Ana Gabriela Fuentes-Nava, et al. This is an open-access article distributed under the terms of the Creative Commons Attribution License, which permits unrestricted use, distribution, and reproduction in any medium, provided the original author and source are credited.

Source of Support: Nil, Conflict of Interest: None declared. 